Conclusions: Greater BMI, FBG, HDL, triglyceride levels and TC/HDL ratio characterised the young adults with pre/mild hypertension. The data suggests that hypertension in young adults is secondary to metabolic syndrome.

\title{
REVERSIBILITY OF THE EFFECTS CAUSED BY FENUGREEK SEEDS AQUEOUS (FSA) EXTRACT ON THE ESTROUS CYCLE AND REPRODUCTIVE HORMONES IN THE RAT ANIMAL MODEL.
}

\author{
A. K. Allow ${ }^{1}$, S. Abdulmogni ${ }^{2}$, N. M Bakrim², N. Hassan ${ }^{3}$ and M. I .A. Mustafa Mahmud ${ }^{3}$ \\ ${ }^{1}$ Department of Basic Medical Sciences (BMS), Faculty of Medicine (FOM), International Islamic University Malaysia (IIUM), \\ Indera Mahkota Campus, 25200 Kuantan, Malaysia. ${ }^{2}$ Physiology Dept., Faculty of Medicine and Health Sciences, Sana'a \\ University, Yemen. ${ }^{3}$ Faculty of Pharmacy, IIUM, Indera Mahkota Campus, 25200 Kuantan, Malaysia.
}

Introduction: In evaluating the potential of fenugreek seeds aqueous (FSA) extract as a contraceptive, it is essential to assess the reversibility of its anti-fertility effects. Reversibility is defined as ability for an induced altered physiological state to return to the normal state.

Objective: The aim of the present work was to evaluate the reversibility of changes in the oestrous cycle and levels of reproductive hormones in female rats following withdrawal treatment of FSA extract.

Methodology: Twenty four mature Sprague Dawley female rats were randomly divided into three groups of 8 rats each. Group A was the control and given distilled water. Group B was treated with $500 \mathrm{mg} / \mathrm{kg} /$ day of FSA extract for 15 days. Group C was the reversibility group in which the female rats were also treated with $500 \mathrm{mg} / \mathrm{kg} / \mathrm{day}$ FSA extract and further observed for 21 days for reversibility effects. Daily vaginal smear cytology was performed and blood samples were taken from all animals after 15 days.

Results: The abnormal oestrous cycles following FSA treatment were gradually returned to normal within the 21 days of observation post treatment withdrawal. Administration of FSA extract led to a decrease in the serum concentration of estrogen $(\mathrm{P}<0.001)$, progesterone $(\mathrm{P}=0.021)$, FSH $(\mathrm{P}=0.416)$ and $\mathrm{LH}(\mathrm{P}=0.381)$ while serum prolactin concentration was significantly increased $(\mathrm{P}<0.001)$. After 15 days of treatment withdrawal, serum estrogen, progesterone, FSH and LH concentration were not significantly different $(\mathrm{P} \geq 0.192)$ in compare to the control group.

Conclusion: Withdrawal of FSA extract treatment restored the abnormal oestrous cycle and reproductive hormones to the normal state.

\section{IS THERE ANY EFFECT (S) OF FENUGREEK SEEDS AQUEOUS EXTRACT ON THE REGULARITY OF ESTROUS CYCLE AND QUANTITY OF OVARIAN FOLLICLES OF FEMALE RATS?}

PhD, A.K.Allow ${ }^{1}$, N. M. Bakrim ${ }^{1}$, Z. Yousef ${ }^{1}$, IM Al-Ani ${ }^{1}$, NM Hassan ${ }^{2}$, M Kubaysi ${ }^{3}$ and MIA, Mustafa ${ }^{1}$.

${ }^{1}$ Department Basic Medical Science (BMS), Faculty of Medicine, International Islamic University Malaysia (IIUM), 25200

Kuantan, Malaysia. ${ }^{2}$ Faculty of Pharmacy, IIUM, 25200 Kuantan, Malaysia. ${ }^{3}$ Obstretric and Gyanecology Department, Faculty of Medicine, IIUM, 25200 Kuantan, Malaysia

Introduction: The presence of diosgenin in fenugreek seeds is believed to be the active compound responsible for fenugreeks anti-fertility property.

Objective: The purpose of this study to evaluate the potential effects of fenugreek seeds aqueous extract (FSA) extract on the regularity of oestrous cycle and quantity of ovarian follicles.

Methodology: Thirty two healthy mature female rats were randomly divided into four groups of 8 rats each. The first group A was the control and received distilled water; the B, C and D received 250, 500 and $1000 \mathrm{mg} / \mathrm{kg} /$ day FSA extract, respectively, for 15 days. Daily vaginal smear cytology was examined and ovaries of the animals were removed after 15 days for histological study. 